\title{
Effect of telemedicine on diabetic treatment and complications
}

\author{
Hamed Saeed Al-Ghamdi ${ }^{1 *}$, Abdelrahman Abdrabou Eldabbari ${ }^{2}$, Salma Awadh Alharthi ${ }^{3}$, \\ Shahad Abdulbasit Alhutami ${ }^{3}$, Mohammed Omar Aljiffry ${ }^{4}$, Sultana Saadaldeen Alsharif ${ }^{5}$, \\ Ahmad Maeesh Alqurashi ${ }^{4}$, Saeed Abdulsattar Mohammed ${ }^{4}$, Maram Adnan Rawah ${ }^{3}$, \\ Mona Saleh Almalki ${ }^{4}$, Taghreed Jaman Alotaibi ${ }^{4}$
}

\author{
${ }^{1}$ Department of Internal Medicine, King Abdulaziz Hospital, Jeddah, Saudi Arabia \\ ${ }^{2}$ Department of Internal Medicine, Dr. Suliman Alhabib Hospital, Riyadh, Saudi Arabia \\ ${ }^{3}$ Department of Internal Medicine, King Fahad Hospital, Jeddah, Saudi Arabia \\ ${ }^{4}$ Department of Internal Medicine, King Abdulaziz Specialist Hospital, Taif, Saudi Arabia \\ ${ }^{5}$ Department of Internal Medicine, Al Thager General Hospital, Jeddah, Saudi Arabia
}

Received: 10 April 2021

Accepted: 17 April 2021

\section{*Correspondence:}

Dr. Hamed Saeed Al-Ghamdi,

E-mail: h.s.alghamdi@hotmail.com

Copyright: ( $)$ the author(s), publisher and licensee Medip Academy. This is an open-access article distributed under the terms of the Creative Commons Attribution Non-Commercial License, which permits unrestricted non-commercial use, distribution, and reproduction in any medium, provided the original work is properly cited.

\begin{abstract}
Investigations have also reported that the efficacy of telemedicine in the easy delivery of information and provision of regular patient-doctor interaction and therefore, enhancing the quality of care of diabetics and reducing the risk of developing severe complications. In the present study, we aimed to discuss telemedicine use in the management of diabetic complications, depending on information from studies within the literature. Overall, telemedicine assists patients to properly maintain basic and adequate levels of blood glucose levels through continuous reminders. Therefore, the application of this modality requires patients to have home-based assessment devices for the regular monitoring of the blood glucose levels. Moreover, the modality also enhances the connection between the patient and the physicians which make the patient at an ease of being able to communicate with the doctor whenever necessary and therefore, this might have a positive psychological impact on these patients. Moreover, telemedicine can provide patients with adequate treatment schedules that might help patients comply with their drugs. Additionally, it can provide the most appropriate dietary components that can also help in achieving the best intervention, with the necessary supervision. Although there is good evidence about the efficacy of telemedicine as an efficacious modality in the treatment of diabetes and management of complications, the evident application in the healthcare settings is still poor. The main reason behind such lack of proper application is the potential lack of adequate financial support, lack of experienced staff dealing with the modalities and maintaining the best quality of care for the patients.
\end{abstract}

Keywords: Diabetes, Telemedicine, Care, Management, Complications

\section{INTRODUCTION}

Reports have shown that diabetes mellitus (DM) nearly affects more than 30 million patients within the United States in 2015 and 415 million globally, which is expected to increase to 642 million by $2040 .{ }^{1}$ The chronic condition mainly has two types, including type 1 (T1DM) and type 2 (T2DM), with two different pathophysiological pictures. In T1DM, which usually occurs in childhood, patients suffer from absolute insulin deficiency, while in T2DM, which usually occurs in the older population, patients usually suffer from insulin resistance and deficiency may usually occur later on. ${ }^{2}$ As 
a result of either of these mechanisms and the subsequent increase in the serum glucose levels or hyperglycemia, many complications can affect many organs within the body and cause life-disabling manifestations that may end up with death. The affected organs might include the cardiovascular organs, kidneys, skin, eyes and nerves. It has been estimated that diabetes-induced mortality is a leading cause of global deaths owing to secondary amputations, kidney failure and blindness. ${ }^{3-5}$ Accordingly diabetic patients are advised to undergo routine checkups for the proper management and interventions against these complications.

The management of DM complications is exhaustive as patients are required to perform many examinations and stick to strict treatment modalities. Based on these challenges, researchers have aimed at developing novel approaches for the easy delivery of the management modalities and to encourage patients to adopt the self-care approach to reduce the burdens of the exhaustive management procedures and thus, enhancing the quality of life for these patients and the quality of care for the diabetic complications.

Among the suggested modalities, telemedicine has been proposed as an efficacious approach that can help achieve these aims. The idea behind this approach is to increase the interaction between the patients and their physicians and enhance the quality of information exchange between the two parties, which therefore, can enhance the quality of care and is cost and effort-effective. ${ }^{6,7}$ Telemedicine has been previously reported in the management of many disorders, to increase the patients' compliance to treatment, as in hypertension and other cardiovascular diseases. ${ }^{8-10}$ In DM, it has been used to routinely followup the status of the affected patients and the regular check-up of their blood glucose levels to obtain the best outcomes. ${ }^{11,12}$ Previous relevant investigations have also reported the efficacy of the modality in the easy delivery of information and provision of regular patient-doctor interaction and therefore, enhancing the quality of care of diabetics and reducing the risk of developing severe complications. $^{13,14}$ In the present study, we aimed to discuss telemedicine use in the management of diabetic complications, depending on information from studies within the literature.

\section{METHODS}

A systematic search was conducted to identify relevant studies in the following databases: pubmed, medline, web of science, embase, google scholar, and scopus. The following search terms were used (telemedicine or telemedicine), (diabetes or diabetes mellitus), (treatment), (effect or outcome) and (complications). The reference lists were manually searched to identify additional relevant studies meeting inclusion criteria. We included any study that reports the telemedicine effect on diabetic treatment or complication. No restrictions were applied.

\section{DISCUSSION}

\section{Telemedicine in diabetic treatment and complications}

Telemedicine has been extensively reported in the literature for many purposes regarding the management of diabetes. Studies showed that previous diabetes management modalities can be used to increase the knowledge about the disease and management modalities, enhance the perception about the disease and the potential complications, strengthening the relationship between the patient and his family and facilitation of the management approaches to increase the compliance to these modalities over the long term. ${ }^{15-22}$ However, many challenges should be considered to alleviate the quality of care. Previous investigations reported that it can overcome the poor access to these modalities in low socio-economic and rural areas might limit the benefits of the modality. Moreover, some studies reported that it is not costefficacious and is associated with poor compliance among diabetic patients. ${ }^{23,24}$ Therefore, telemedicine has been proposed as an efficacious modality for the management of complications and treatment of diabetes. ${ }^{25}$ Currie et al also reported that clinical and face-to-face regular assessments might be potentially better than telemedicine because patients that usually comply less with the medical regimens are more likely to adhere to this new modality and therefore, continuous guiding is encouraged. ${ }^{26}$ Therefore, telemedicine applications should be carefully approached on a regular basis.

\section{Self-monitoring and assessment of complications}

The main of applying telemedicine modalities is to provide the patient with the necessary information about the best ways to achieve the prevention and prevent the possible complications and to enhance the frequency of communication between the patient and his doctor. As advances have emerged within these modalities, innovative approaches have been created to facilitate the modality and obtain the best clinical outcomes. Previous studies have demonstrated that mobile-based interventions are promising and efficacious modalities in obtaining good interventional outcomes and improving the quality of life for the potential patients that have been using them. ${ }^{27,28}$ Based on the findings of these various investigations, mobile-based interventions for the treatment and management of diabetic complications are currently validated for the easy delivery and enhancement of these outcomes. ${ }^{29,30}$ However, the availability of mobile phones and the ability to afford them by many individuals might be a potential holdback in achieving these outcomes, as previous research reported that $9 \%$ and $22 \%$ of the adults and adolescents, respectively, cannot have mobile phones and therefore, the modality is not applicable for this large proportion of the population. Besides, evidence from previous studies also showed that applying telemedicine for children and adolescents was not significantly associated with any improvements in the primary outcomes of glycemic control, including 
maintaining normal blood glucose levels, although it has been noticed that the modality was associated with increased adherence and compliance, in addition to the increased knowledge and communication between the patients, families, and doctors. ${ }^{31-33}$ Therefore, future investigations should be continued and should focus on addressing these challenges to furtherly enhance the outcomes.

To overcome the limited availability of mobile phones among adults and adolescents, previous investigations have proposed internet-based interventions as an alternative modality as the availability of internet among the population is more common than mobile phones within the United States. Moreover, another reason that may favor the use of internet-based approaches is the easy-accessibility to diabetes-related information, especially in the younger population. ${ }^{34}$ Previous investigations have reported a significant enhancement in all aspects of glycemic control and management of diabetic complications and related adverse events in both adults and children. ${ }^{35-40}$ However, it should be noted that internet-based approaches also face some challenges that might limit the results. The main challenge is the less frequent access to the internet and the limited time spent on it, as reported by previous investigations that reported a variable rate of participation in the intervention programs that ranges between $11.5 \%$ and $37 \% .^{36,39}$

Self-management interventional approaches for DM are now validated and efficacious modalities with variablerelated techniques that can be applied to suit the patients' needs and circumstances. These include text messaging, web-based portals, internet texting and e-mails, voice calls, conducting video conferences and seminars. ${ }^{41,42}$ The previous meta-analysis by Holtz et al.,30 which included articles before 2011 reported that most of the included articles showed that telemedicine application in the treatment and management of diabetic complications was effective in obtaining favorable outcomes, as per the findings from 21 articles that investigated patients with T1DM and other with T2DM. Another meta-analysis by Farmer et al which was published in 2005 also reported that in addition to being efficacious in managing diabetic complications and enhancing compliance to treatment, telemedicine approaches are also feasible for the patient and the doctor and are time and cost-effective. ${ }^{13}$ In 2019, another scoping review was conducted by Borris et al to discuss the effect of telemedicine on self-management and clinical complications in patients with T1DM and T2DM. ${ }^{43}$ The authors managed to include 45 articles from the literature, however, they depended on only 10 of them to discuss the relevant outcomes. The authors reported that based on the most commonly assessed modality in these studies (HbA1c levels), telemedicine application was associated with enhanced self-management behaviors and enhanced clinical outcomes. However, it appears that neither of the included studies assessed the long-term complications of diabetes and the effect of telemedicine on reducing the risk of developing organ damage and mortality.

Overall, telemedicine assists patients to properly maintain basic and adequate levels of blood glucose levels through continuous reminders. Therefore, the application of this modality requires patients to have home-based assessment devices for the regular monitoring of the blood glucose levels. Moreover, the modality also enhances the connection between the patient and the physicians which make the patient at an ease of being able to communicate with the doctor whenever necessary, and therefore, this might have a positive psychological impact on these patients. Moreover, telemedicine can provide patients with adequate treatment schedules that might help patients comply with their drugs. Additionally, it can provide the most appropriate dietary components that can also help in achieving the best intervention, with the necessary supervision.

The previous scoping review by Borris et al reported that although no significance was found in the findings, studies showed that improved clinical and physical outcomes were associated with the application of telemedicine devices in diabetes care. ${ }^{43}$ Moreover, the blood glucose and lipid profiles were significantly enhanced in these patients. On the other hand, Borris et al reported that the effect on vascular diseases and longterm complications was not clear at this time due to the lack of evidence and long-term investigations that reported this outcome. ${ }^{43}$ Moreover, it is worth mentioning that the telemedicine modalities might be also limited to the lack of adequate examination by the doctors. These are only useful in the monitoring of the blood glucose levels and reporting of the main physical complications and adverse events. However, complex procedures and clinical assessment modalities are usually limited as a result of the limited face-to-face interactions between the doctor and physician. Therefore, most studies in the literature are not directed to assess such outcomes, which might be illogical to be assessed for judging the efficacy of telemedicine that has been designed for another purpose.

\section{Current challenges for telemedicine in diabetes}

Although there is good evidence about the efficacy of telemedicine as an efficacious modality in the treatment of diabetes and management of complications, the evident application in the healthcare settings is still poor. The main reason behind such lack of proper application is the potential lack of adequate financial support, lack of experienced staff dealing with the modalities and maintaining the best quality of care for the patients. Financial compensations for these services have been a huge obstacle for the providers of the service since it was suggested. ${ }^{44,45}$ Another obstacle for using telemedicine in healthcare settings is the legal issues that require solid paperwork and many ethical considerations before it was legalized. ${ }^{44}$ Moreover, the attrition of patients that are 
supposed to engage in making the modality successful, has also been reported to be low among studies in the literature as previously mentioned. ${ }^{36,39}$ Additionally, even in patients that have been reported to engage in the procedures of telemedicine, the compliance was not adequate due to the lack of adequate necessary face-toface supervision. This was supported by a previous review by Guljas et al that reported that patients, that were being followed using telemedicine modalities, did not adequately upload the supposedly-regularly assessed blood glucose levels to the system for maintaining adequate regular monitoring procedures. ${ }^{46}$

\section{CONCLUSION}

In the present study, we have reviewed the literature to study the effect of telemedicine on diabetic treatment and complications. Self-management interventional approaches for DM are now validated and efficacious modalities with variable-related techniques that can be applied to suit the patients' needs and circumstances. These include text messaging, web-based portals, internet texting and e-mails, voice calls, conducting video conferences and seminars. Although there was good evidence about the efficacy of telemedicine as an efficacious modality in the treatment of diabetes and management of complications, the evident application in the healthcare settings was still poor. The main reason behind such lack of proper application was the potential lack of adequate financial support, lack of experienced staff dealing with the modalities and maintaining the best quality of care for the patients.

\section{Funding: No funding sources}

Conflict of interest: None declared

Ethical approval: Not required

\section{REFERENCES}

1. Ogurtsova K, Fernandes JDDR, Huang Y, Linnenkamp U, Guariguata L, Cho NH, et al. IDF Diabetes Atlas: Global estimates for the prevalence of diabetes for 2015 and 2040. Diabetes Res Clin Pract. 2017;128:40-50.

2. Association AD. Diagnosis and classification of diabetes mellitus. Diabetes Care. 2010;33(1):62-9.

3. Sasaki A, Horiuchi N, Hasegawa K, Uehara M. Mortality and causes of death in type 2 diabetic patients: a long-term follow-up study in Osaka district, Japan. Diabetes Res Clin Pract. 1989;7(1):33-40.

4. Zhu X, Zhang Y, Bai L, Zhang X, Zhou J, Yang J. Prediction of risk of diabetic retinopathy for allcause mortality, stroke and heart failure. Medicine. 2017;96(3):5894.

5. Asfandiyarova NS. Risk factors of death in diabetes mellitus. Klinicheskaia Meditsina. 2016;94(9):697700.

6. Craig J, Petterson V. Introduction to the practice of telemedicine. J Telemed Telecare. 2005;11(1):3-9.
7. Balkhi AM, Reid AM, Westen SC, Olsen B, Janicke DM, Geffken GR. Telehealth interventions to reduce management complications in type 1 diabetes: a review. World J Diabetes. 2015;6(3):371-9.

8. Omboni S, Caserini M, Coronetti C. Telemedicine and M-Health in hypertension management: technologies, applications and clinical evidence. High Blood Press Cardiovasc Prev. 2016;23(3):18796.

9. Caldarola P, Gulizia MM, Gabrielli D, Sicuro M, Gennaro LD, Giammaria M, et al. ANMCO/SIT Consensus Document: telemedicine for cardiovascular emergency networks. European Heart J Suppl. 2017;19:229-43.

10. Eberly LA, Khatana SAM, Nathan AS, Snider C, Julien HM, Deleener ME, et al. Telemedicine outpatient cardiovascular care during the COVID-19 pandemic: bridging or opening the digital divide? Circulation. 2020;142(5):510-2.

11. Ahring KK, Ahring JPK, Joyce C, Farid NR. Telephone modem access improves diabetes control in those with insulin-requiring diabetes. Diabetes Care. 1992;15(8):971-5.

12. Bergenstal RM, Anderson RL, Bina DM, Johnson ML, Davidson JL, Solarz-Johnson B, et al. Impact of modem-transferred blood glucose data on clinician work efficiency and patient glycemic control. Diabetes Technol Ther. 2005;7(2):241-7.

13. Farmer A, Gibson OJ, Tarassenko L, Neil A. A systematic review of telemedicine interventions to support blood glucose self-monitoring in diabetes. Diabet Med. 2005;22(10):1372-8.

14. Liang X, Wang Q, Yang X, Cao J, Chen J, Mo X, et al. Effect of mobile phone intervention for diabetes on glycaemic control: a meta-analysis. Diabet Med. 2011;28(4):455-63.

15. Heidgerken AD, Merlo L, Williams LB, Lewin AB, Gelfand $\mathrm{K}$, <alasanos $\mathrm{T}$, et al. Diabetes awareness and reasoning test: A preliminary analysis of development and psychometrics. Child Healthcar. 2007;36(2):117-36.

16. Murphy HR, Rayman G, Skinner TC. Psychoeducational interventions for children and young people with Type 1 diabetes. Diabet Med. 2006;23(9):935-43.

17. Borus JS, Laffel L. Adherence challenges in the management of type 1 diabetes in adolescents: prevention and intervention. Curr Opin Pediatr. 2010;22(4):405-11.

18. Knight K, Bundy C, Morris R, Higgs JF, Jameson $\mathrm{RA}$, Unsworth $\mathrm{P}$, et al. The effects of group motivational interviewing and externalizing conversations for adolescents with Type-1 diabetes. Psychol Heal Med. 2003;8(2):149-57.

19. Wysocki T, Harris MA, Buckloh LM, Mertlich D, Lochrie AS, Taylor A, et al. Effects of behavioral family systems therapy for diabetes on adolescents' family relationships, treatment adherence, and metabolic control. J Pediatr Psychol. 2006;31(9):928-38. 
20. Wysocki T, Harris MA, Greco P, Bubb J, Danda CE, Harvey LM, et al. Randomized, controlled trial of behavior therapy for families of adolescents with insulin-dependent diabetes mellitus. J Pediatr Psychol. 2000;25(1):23-33.

21. Adolfsson P, Veijola R, Huot C, Hansen HD, Lademann JB, Phillip M. Safety and patient perception of an insulin pen with simple memory function for children and adolescents with type 1 diabetes-the REMIND study. Curr Med Res Opin. 2012;28(9):1455-63.

22. Korytkowski M, Bell D, Jacobsen C, Suwannasari R. A multicenter, randomized, open-label, comparative, two-period crossover trial of preference, efficacy, and safety profiles of a prefilled, disposable pen and conventional vial/syringe for insulin injection in patients with type 1 or 2 diabetes mellitus. Clin Ther. 2003;25(11):2836-48.

23. Griffin SJ. Lost to follow-up: the problem of defaulters from diabetes clinics. Diabet Med. 1998;(3):14-24.

24. Shore JH, Brooks E, Savin DM, Manson SM, Libby AM. An economic evaluation of telehealth data collection with rural populations. Psychiatr Serv. 2007;58(6):830-5.

25. Russell-Minda E, Jutai J, Speechley M, Bradley K, Chudyk A, Petrella R. Health technologies for monitoring and managing diabetes: a systematic review. J Diabetes Sci Technol. 2009;3(6):1460-71.

26. Currie CJ, Peyrot M, Morgan CL, Poole CD, Jenkins-Jones S, Rubin RR, et al. The impact of treatment non-compliance on mortality in people with type 1 diabetes. J Diabetes Complications. 2013;27(3):219-23.

27. Shulman RM, O'Gorman CS, Palmert MR. The impact of telemedicine interventions involving routine transmission of blood glucose data with clinician feedback on metabolic control in youth with type 1 diabetes: a systematic review and metaanalysis. Int J Pediatr Endocrinol. 2010;2010:1-9.

28. Howe CJ, Jawad AF, Tuttle AK, Moser JT, Preis C, Buzby $M$, et al. Education and telephone case management for children with type 1 diabetes: a randomized controlled trial. J Pediatr Nurs. 2005;20(2):83-95.

29. Bin-Abbas B, Jabbari M, Al-Fares A, El-Dali A, AlOrifi F. Effect of mobile phone short text messages on glycaemic control in children with type 1 diabetes. J Telemed Telecare. 2014;20(3):153-6.

30. Holtz B, Lauckner C. Diabetes management via mobile phones: a systematic review. Am Telemed Assoc. 2012;18(3):175-84.

31. Hauser ST, Jacobson AM, Lavori P, Wolfsdorf JI, Herskowitz RD, Milley JE, et al. Adherence among children and adolescents with insulin-dependent diabetes mellitus over a four-year longitudinal follow-up: II. Immediate and long-term linkages with the family milieu. J Pediatr Psychol. 1990;15(4):527-42.
32. Cafazzo JA, Casselman M, Hamming N, Katzman DK, Palmert MR. Design of an mHealth app for the self-management of adolescent type 1 diabetes: a pilot study. J Med Inter Res. 2012;14(3):70.

33. Louch G, Dalkin S, Bodansky J, Conner M. An exploratory randomised controlled trial using short messaging service to facilitate insulin administration in young adults with type 1 diabetes. Psychol Health Med. 2013;18(2):166-74.

34. Daniilidou K, Triantafyllou P, Resta M, Dimitriadou M, Christoforidis A. Level of internet use among Greek adolescents with type 1 diabetes. Int $\mathbf{J}$ Adolesc Med Health. 2017;31(1).

35. Grey M, Whittemore R, Jeon S, Murphy K, Faulkner MS, Delamater A. Internet psychoeducation programs improve outcomes in youth with type 1 diabetes. Diabetes Care. 2013;36(9):2475-82.

36. Mulvaney SA, Rothman RL, Wallston KA, Lybarger C, Dietrich MS. An internet-based program to improve self-management in adolescents with type 1 diabetes. Diabetes Care. 2010;33(3):602-4.

37. Shalitin S, Ben-Ari T, Yackobovitch-Gavan M, Tenenbaum A, Lebenthal Y, Vries LD, et al. Using the internet-based upload blood glucose monitoring and therapy management system in patients with type 1 diabetes. Acta Diabetol. 2014;51(2):247-56.

38. Toma $\mathrm{T}$, Athanasiou $\mathrm{T}$, Harling L, Darzi A, Ashrafian H. Online social networking services in the management of patients with diabetes mellitus: systematic review and meta-analysis of randomised controlled trials. Diabetes Res Clin Pract. 2014;106(2):200-11.

39. Azar M, Gabbay R. Web-based management of diabetes through glucose uploads: has the time come for telemedicine? Diabetes Res Clin Pract. 2009;83(1):9-17.

40. Ralston JD, Hirsch IB, Hoath J, Mullen M, Cheadle A, Goldberg HI. Web-based collaborative care for type 2 diabetes: a pilot randomized trial. Diabetes Care. 2009;32(2):234-9.

41. Izquierdo R, Morin PC, Bratt K, Moreau Z, Meyer $\mathrm{S}$, Ploutz-Snyder R, et al. School-centered telemedicine for children with type 1 diabetes mellitus. J Pediatr. 2009;155(3):374-9.

42. Hanauer DA, Wentzell K, Laffel N, Laffel LM. Computerized automated reminder diabetes system (CARDS): e-mail and SMS cell phone text messaging reminders to support diabetes management. Diabetes Technol Ther. 2009;11(2):99-106.

43. Borries T, Dunbar A, Bhukhen A, Rismany J, Kilham J, Feinn R, et al. The impact of telemedicine on patient self-management processes and clinical outcomes for patients with Types I or II diabetes mellitus in the United States: a scoping review. Diabetes Metab Syndr. 2019;13(2):1353-7.

44. Soares NS, Langkamp DL. Telehealth in developmental-behavioral pediatrics. J Development Behavior Pediatr. 2012;33(8):656-65. 
45. Whitten P, Buis L. Private payer reimbursement for telemedicine services in the United States. Am Telemed Assoc. 2007;13(1):15-23.

46. Guljas R, Ahmed A, Chang K, Whitlock A. Impact of telemedicine in managing type 1 diabetes among school-age children and adolescents: an integrative review. J Pediatr Nurs. 2014;29(3):198-204.
Cite this article as: Al-Ghamdi HS, Eldabbari AA, Alharthi SA, Alhutami SA, Aljiffry MO, Alsharif SS, et al. Effect of telemedicine on diabetic treatment and complications. Int J Community Med Public Health 2021;8:2582-7. 